\title{
A new strategy for assessing selenoprotein function: siRNA knockdown/knock-in targeting the $3^{\prime}$ '-UTR
}

\author{
MIN-HYUK YOO, ${ }^{1}$ XUE-MING XU, ${ }^{1}$ ANTON A. TURANOV, ${ }^{2}$ BRADLEY A. CARLSON, ${ }^{1}$ \\ VADIM N. GLADYSHEV, ${ }^{2}$ and DOLPH L. HATFIELD ${ }^{1}$ \\ ${ }^{1}$ Molecular Biology of Selenium Section, Laboratory of Cancer Prevention, Center for Cancer Research, National Institutes of Health, \\ Bethesda, Maryland 20892, USA \\ ${ }^{2}$ Department of Biochemistry, University of Nebraska, Lincoln, Nebraska 68588, USA
}

\begin{abstract}
Selenocysteine insertion into protein in mammalian cells requires RNA elements in the $\mathbf{3}^{\prime}$-untranslated regions (3'-UTRs) of selenoprotein genes. The occurrence of these conserved sequences should make selenoproteins particularly amenable for knockdown/knock-in strategies to examine selenoprotein functions. Herein, we utilized the 3'-UTR of various selenoproteins to knock down their expression using siRNAs and then knock in expression using constructs containing mutations within the target region. Thioredoxin reductase 1 (TR1) knockdown in a mouse kidney cell line resulted in the cells growing about $10 \%$ more slowly, being more sensitive to UV radiation, and having increased apoptosis in response to UV than control cells. The knockdown cells transfected with a construct encoding the wild-type TR1 gene and having mutations in the sequences targeted by siRNA restored TR1 expression and catalytic activity, rendered the knockdown cells less sensitive to UV, and protected the cells against apoptosis. We also applied this technique to other selenoproteins, selenophosphate synthetase 2 and glutathione peroxidase 1, and found that mRNA and protein levels were restored following transfection of knockdown cells with the corresponding knock-in constructs. In addition to important new insights into the functions of key mammalian selenoproteins, the data suggest that the RNAi-based knock-in technology could distinguish phenotypes due to off-targeting and provide a new method for examining many of the subtleties of selenoprotein function not available using RNAi technology alone.
\end{abstract}

Keywords: glutathione peroxidase 1; knockdown/knock-in; siRNA; selenophosphate synthetase 2; thioredoxin reductase 1

\section{INTRODUCTION}

The targeted removal of protein expression using RNAi technology has become an extremely powerful tool for studying protein function (e.g., see Hannon 2002; Novina and Sharp 2004). However, a serious deficiency in this technology has been that it could only achieve a decreased expression of a targeted gene product, limiting its application for an in-depth analysis of protein function. To overcome this deficiency, several laboratories have developed gene replacement strategies that involve different approaches in developing knockdown (targeting) and knock-in (replacement) vectors. For example, silent mutations in the knock-in vector that take advantage of the genetic code's degeneracy have been used to circumvent the knockdown vector (Kim and Rossi 2003; Jiang and

Reprint requests to: Dolph L. Hatfield, Molecular Biology of Selenium Section, Laboratory of Cancer Prevention, Center for Cancer Research, Building 37, Room 6032, National Institutes of Health, Bethesda, MD 20892, USA; e-mail: hatfield@mail.nih.gov; fax: (301) 435-4957.

Article published online ahead of print. Article and publication date are at http://www.rnajournal.org/cgi/doi/10.1261/rna.533007.
Price 2004; Kiang et al. 2005). Species variations in gene sequences between the knockdown, host protein and the knock-in, homologous (from a different species with a varying sequence in the target site) protein have also been used in gene replacement strategy (Puthenveedu and Linstedt 2004; Unwalla et al. 2006; Yu et al. 2006). Other approaches such as "differential RNA interference" (Laatsch et al. 2004) and simultaneous microinjection of knockdown/knock-in RNAs into Xenopus oocytes (Jallow et al. 2004) have also been used as gene replacement strategies. It should also be noted that replacement of protein expression in mammalian cells by knocking in mRNA synthesis following its knockout is an established method of studying protein function in the absence of the host endogenous protein, but this approach requires the initial generation of knockout and transgenic animals and is difficult to apply to essential genes (e.g., see Carlson et al. 2005). The ability to combine RNAi-based knockdown and knock-in approaches is extremely useful and is far simpler than gene replacement studies involving mouse models.

A unique feature of the mRNAs encoding selenocysteine $(\mathrm{Sec})$-containing proteins is that they require a stem-loop 
structure known as a Sec insertion sequence (SECIS) element for insertion of Sec into the nascent protein in response to a UGA codon (Low and Berry 1996). SECIS elements are located in the $3^{\prime}$-untranslated region $\left(3^{\prime}\right.$ UTR) of eukaryotic selenoprotein mRNAs. The highly developed 3'-UTR affords an opportunity of knocking down and knocking in their expression without involving the coding region. There are 25 known selenoproteins in humans and 24 in rodents (Kryukov et al. 2003), and these proteins have diverse cellular functions, most of which are unknown. Furthermore, there has been considerable interest in this unique, selenium-containing class of proteins in recent years as these proteins are thought to be largely responsible for the many health benefits attributed to the trace element selenium. For example, selenoproteins have roles in cancer and heart disease prevention, in viral inhibition, and roles in development, male reproduction, and slowing the aging process (Hatfield et al. 2006). Therefore, the development of simple strategies to elucidate their functions is important to providing a better understanding of the role of selenium in health.

Among the 24 selenoproteins in rodents (Kryukov et al. 2003), there are three mammalian thioredoxin reductases, of which one, thioredoxin reductase 1 (TR1), is an essential Sec-containing oxidoreductase that controls the redox state of cytosolic thioredoxin and is one of the major redox regulators in mammalian cells (Mustacich and Powis 2000; Rundlof and Arner 2004). Herein, we used this protein as a model in developing a siRNA knockdown-mRNA knock-in strategy for studying selenoprotein function. We then applied this technique to other selenoproteins, selenophosphate synthetase 2 (SPS2) and glutathione peroxidase 1
(GPx1), to demonstrate that this approach could have wide use in studying selenoprotein function.

\section{RESULTS}

\section{siRNA constructs and TR1 mRNA knockdown}

Four siRNA constructs targeting the $3^{\prime}$-UTR of mouse TR1, designated siTR1-1-4, were prepared. TCMK-1 cells were transiently transfected with each construct and with the pU6-m3 control construct. The resulting transfected cells were labeled with ${ }^{75} \mathrm{Se}$ to evaluate their ability to knock down TR1 expression. siTR1-3 and siTR1-4, which targeted sites downstream of the SECIS element, were effective in down-regulating TR1 expression, whereas the other two constructs that targeted sites upstream of the SECIS element were ineffective (Fig. 1A).

TMCK-1 cells were then stably transfected with the siTR13, siTR1-4, or control construct and the levels of TR1 mRNA examined by Northern blotting (Fig. 1B). TR1 mRNA levels were substantially reduced in both siTR1 transfected cell lines compared to cells transfected with the control construct. It should be noted that TMCK-1 cells, stably transfected with the siTR1-3 construct, grew $\sim 10 \%$ more slowly than their control cell counterpart, but $\sim 15 \%-20 \%$ faster than cells that were transfected with the siTR1-4 construct (data not shown). The siTR1-3 construct was, therefore, used in all subsequent experiments. siTR1-3 transfected cells and control cells were then labeled with ${ }^{75} \mathrm{Se}$. Knockdown of TR1 mRNA by siTR1-3 resulted in the virtual loss of TR1 expression whereas the expression of other major selenoproteins did not appear to be affected (Fig. 1C).
$\mathbf{A}$

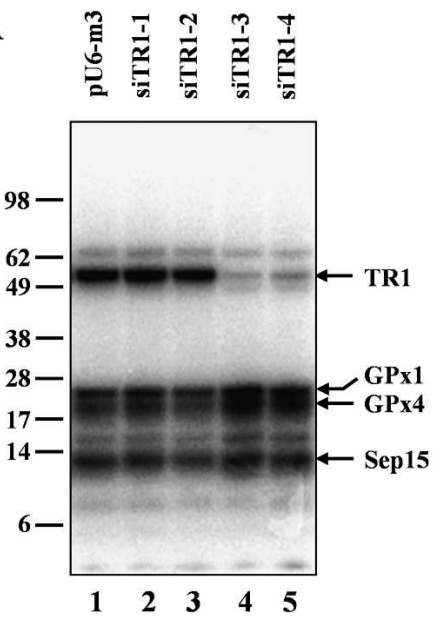

B

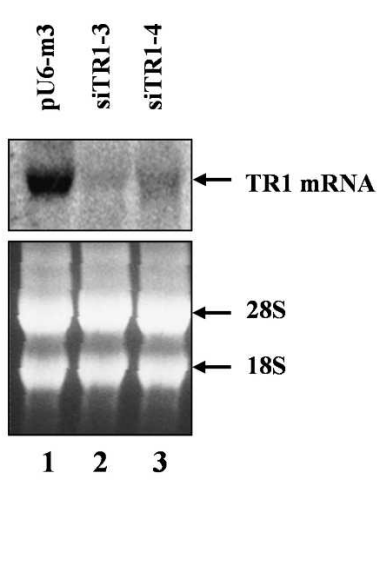

C

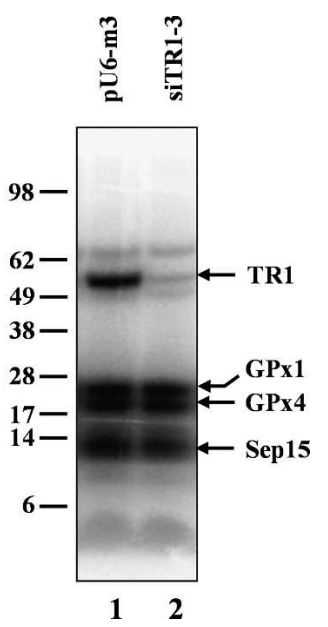

FIGURE 1. Knockdown of TR1 mRNA and TR1. TMCK-1 cells were transfected with the four TR1 siRNA and control constructs either transiently $(A)$ or stably $(B, C)$ and the resulting transfected cells labeled with ${ }^{75} \mathrm{Se}(A, C)$ or analyzed by Northern blotting $(B)$ as follows: $(A$, lane 1), pU6-m3; (lane 2) siTR1-1; (lane 3) siTR1-2; (lane 4) siTR1-3; and (lane 5) siTR1-4. (B, lane 1) pU6-m3; (lane 2) siTR1-3; and (lane 3) siTR14. (C, lane 1) pU6-m3; (lane 2) siTR1-3; and (lane 3) siTR1-4. Major selenoproteins are indicated on the right side and molecular weight markers on the left side of $A$ and $C$, and migration of $18 \mathrm{~S}$ and $28 \mathrm{~S}$ ribosomal RNAs, used as loading controls, are indicated on the right side of the lower portion in $B$. 
It should be noted that GPx1, GPx4, and possibly Sep15, appeared to be slightly enriched in the TR1 knockdown cells relative to control cells transiently transfected with siTR1-3 and siTR1-4 (see Fig. 1A), but a similar enrichment was not observed in the corresponding stably transfected cells (see Fig. 1C). This difference in selenoprotein synthesis may be due, at least in part, to the fact that stably transfected cells have a longer time span to adjust to selenoprotein metabolism in response to TR1 knockdown than transiently transfected cells. Furthermore, the loss of the more abundantly expressed TR1 may result in greater accessibility of other selenoprotein mRNAs to the protein synthesis machinery in the shorter time span found in transiently transfected cells. However, a similar situation may not exist in stably transfected cells due to adjustment of the protein synthesis machinery in maintaining the expression of selenoproteins at their required levels for meeting cellular needs.

\section{Characteristics of TR1 knockdown cells}

Phenotypic changes between control and transfected cell lines were initially tested by examining their responses to UV irradiation. Normally, mammalian cells are treated with $50-100 \mathrm{~J} / \mathrm{m}^{2}$ to induce apoptosis, but we exposed TMCK-1 cells stably transfected with the siTR1-3 or control constructs to less than half this amount of radiation
(30 J/m ${ }^{2}$ ). UV irradiation was selected as a means of examining TR1 function since UV induces reaction oxygen species (ROS) in cells and TR1 is one of the major antioxidants that protects cells against ROS by maintaining thioredoxin in the reduced state. The siTR1-3, TR1 knockdown cells were more sensitive to UV irradiation than the control cells at this level of exposure. Less than $50 \%$ of the TR1 knockdown cells were recovered upon UV irradiation compared to control cells (Fig. 2A). Cleavage of caspase- 3 is an indication of apoptosis and JNK1 and JNK2 are major kinases involved in the apoptosis pathway. We therefore examined the cleavage of caspase- 3 and the phosphorylation of $\mathrm{JNK} 1 / 2$ by Western blot analysis in cells either exposed or unexposed to UV irradiation (Fig. 2B,C, respectively). Untreated control and siTR1-3 cells did not show any cleavage of caspase-3, and control cells treated with UV irradiation had only a slightly visible cleaved caspase-3 band, whereas the siTR1-3 UV-treated cells manifested a clearly visible cleaved caspase-3 (Fig. 2B).

Phosphorylation of JNK1/2 was examined in control pU6-m3 and siTR1-3 transfected cells at 0, 10, and $30 \mathrm{~min}$ after exposure to UV (Fig. 2C). Control cells manifested phosphorylation of JNKs at $30 \mathrm{~min}$ after exposure, but the level was lower than that observed with siTR1-3 transfected cells (designated P-JNK1 in Fig. 2C). Furthermore, both JNK1 and JNK2 were phosphorylated at $30 \mathrm{~min}$ in siTR1-3 transfected cells (designated P-JNK2 in Fig. 2C).
A

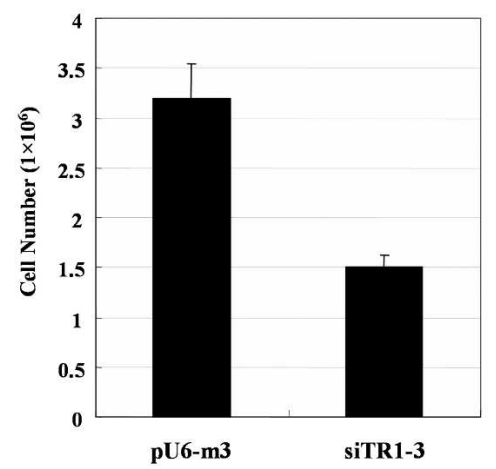

B

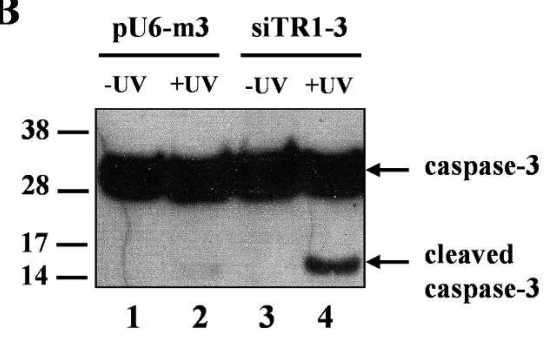

C

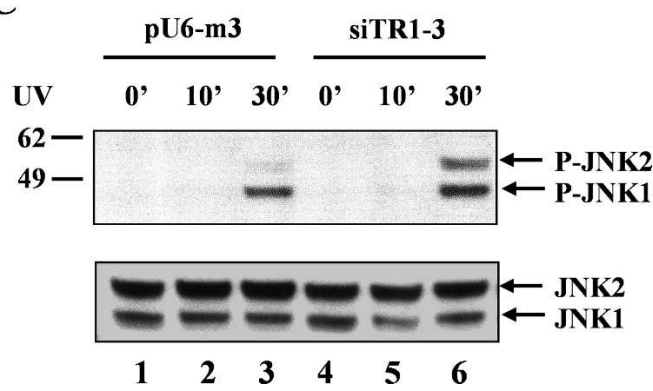

FIGURE 2. UV sensitivity of the siTR1-3 cells. (A) Viability of cells was determined by counting cell numbers after harvesting $24 \mathrm{~h}$ following UV irradiation (see Materials and Methods) of pU6-m3 control cells and siTR1-3 stably transfected cells. (B) Cleavage of caspase-3 was determined by Western blot analysis in cell lysates of UV-irradiated cells: (lanes 1,2) untreated and UV treated pU6-m3 transfected cells; (lanes 3,4) untreated and UV treated siTR1-3 transfected cells, respectively. $(C)$ Phosphorylation of JNK (designated P-JNK1/2) was monitored in pU6-m3 and siTR1-3 cell lysates by Western blot analysis after UV irradiation at 0,10 , and 30 min of pU6-m3 transfected cells (lanes 1-3) and siTR1-3 transfected cells (lanes 4-6). Total amount of JNK is shown as a quantitative control in the lower panel of $C$. Molecular weight markers are indicated on the left in $B$ and $C$. 


\section{TR1 replacement}

TMCK-1 cells that had been stably transfected with the siTR1-3 construct were transiently transfected with the expression vectors encoding either the wild-type (TR1wt) or mutant (siTR1-3m or siTR1-3m $\Delta$ ) mRNAs. Initially, the TR1 mRNA levels were examined in each cell line. Cells stably transfected with pU6-m3 or siTR1-3 were used as controls showing that TR1 mRNA was expressed (Fig. 3A, lane 1) and knocked down (Fig. 3A, lane 2) when these cell lines were transfected transiently. siTR1-3 cells transiently transfected with the TR1wt construct had substantially reduced amounts of TR1 mRNA (Fig. 3A, lane 3 ), while those transfected with TR1-3m appeared to express TR1 mRNA, but the Northern band was smeared, suggesting that it was degraded (Fig. 3A, lane 4). Cells transiently transfected with the siTR $1-3 \mathrm{~m} \Delta$ construct that encoded, in addition to the mutations in the siTR 1 target site, a deletion of the ARE region (Fig. 3A, lane 5) expressed TR1 mRNA.
The above cell lines were labeled with ${ }^{75}$ Se. The pU6-m3 control cell line and that transiently transfected with siTR1$3 \mathrm{~m} \Delta$ had the highest TR1 synthesis (Fig. 3B). Comparison of Figure 3B, lanes 4 and 5, demonstrate the requirement for the ARE deletion in TR1 mRNA for generating an efficient knock-in construct (Gasdaska et al. 1999). The ARE region in the $3^{\prime}$-UTR of TR 1 mRNA is known to regulate mRNA stability, and deletion of this region results in increased mRNA stability and enhanced protein expression (Gasdaska et al. 1999).

\section{Characteristics of TR1 replacement cells}

TR1 activities and expression levels were determined in knock-in and control cells by direct assay and Western blot analysis (Fig. 3C,D, respectively). The knock-in cells manifested higher TR activity than their counterparts transiently transfected with the pcDNA3.1 vector, demonstrating that TR1 had been replaced in the knock-in cells (Fig. 3C).
A

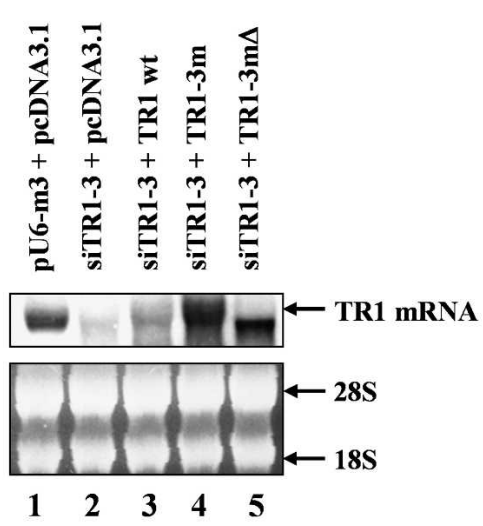

B

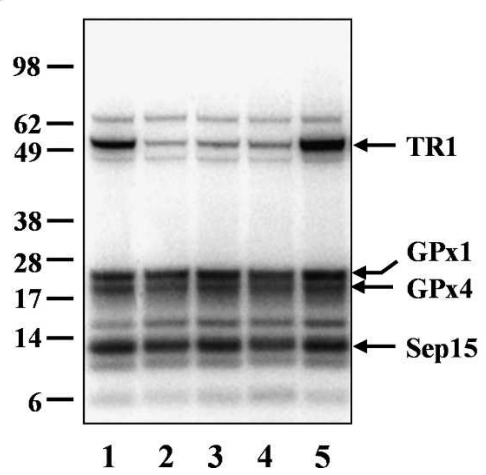

C

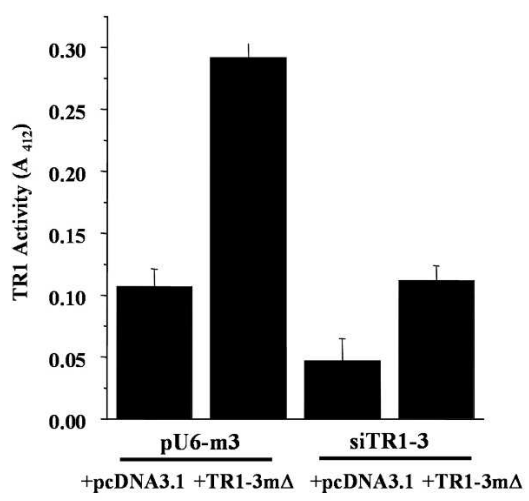

D

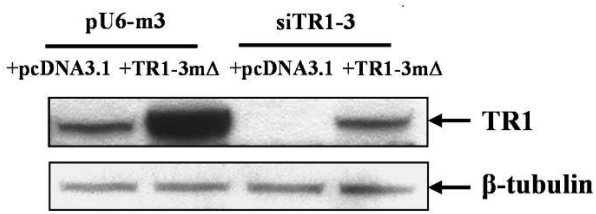

FIGURE 3. TR1 replacement. Cells that were stably transfected with pU6-m3 or siTR1-3 constructs were transiently transfected with the expression vector pcDNA3.1 or the expression vector encoding TR1wt, TR1-3m, or TR1-3m $\Delta$ and the resulting cells analyzed as follows: $(A)$ Northern blotting of RNA extracts to determine TR1 mRNA levels (upper panel): (lane 1) pU6-m3 + pcDNA3.1; (lane 2) siTR1-3 + pcDNA3.1; (lane 3) siTR1-3 + TR1wt; (lane 4) siTR1-3 + TR1-3m; and (lane 5) siTR1-3 + TR1-3ms. Ribosomal 18S and 28S RNA are shown as loading controls (lower panel); (B) Expression of ${ }^{75}$ Se-selenoproteins in extracts from labeled cells: (lane 1) pU6-m3 + pcDNA3.1; (lane 2) siTR1-3 + pcDNA3.1; (lane 3) siTR1-3 + TR1wt; (lane 4) siTR1-3 + TR1-3m; and (lane 5) siTR1-3 + TR1-3m $\Delta$. Molecular weight markers and selenoprotein designations are indicated as given in the legend of Figure 1; $(C)$ Measurement of TR1 activity in cell extracts from pU6-m3 + pcDNA3.1, pU6-m3 + TR1-3m $\Delta$, siTR1-3 + pcDNA3.1, and siTR1-3 + TR1-3m $\Delta$ are as shown, and the data are the mean \pm S.D. $(n=3) ;(D)$ Western blotting of protein extracts from pU6-m3 + pcDNA3.1, pU6-m3 + TR1-3m $\Delta$, siTR1-3 + pcDNA3.1, and siTR1-3 + TR1-3m $\Delta$ assessing the presence of TR1 (upper panel) and using $\beta$-tubulin as an internal control (lower panel). 
The "background" activity in the TR1 knockdown cells transiently transfected with pcDNA3.1 vector is most likely due to TR3 activity and possibly residual TR1 activity, as the enzymatic assay for TR does not distinguish between the different homologs. The amount of TR activity was similar in the pU6-m3 control cells as in the knock-in cells, but was $>2.5$-fold higher in the pU6-m3 cells transiently transfected with the expression TR1wt construct. Western blot analysis confirmed the expression of TR1 in the knock-in cells and reflected the amount of TR 1 in each cell line that was expected from the enzymatic activity assays (see Fig. 3D).

UV-mediated phosphorylation levels of JNK1 and JNK2 in cells stably transfected with the knockdown vector and transiently transfected with either the pcDNA3.1 vector or the knock-in construct were examined. The bands corresponding to phosphorylated JNK1 and JNK2 appeared more intense in the UV-treated cells transiently transfected with pcDNA3.1 than the knock-in cell line, suggesting that TR1 replacement at least partially rescued the cells from UV-mediated apoptosis (Fig. 4A). The intensities of phosphorylated JNK1 and JNK2 are shown relative to the intensities of phosphorylated JNK1 and JNK2 observed in UV-treated control cells when the latter two bands were assigned a value of 1.0 (Fig. 4B), which clearly show that after TR1 knock-in, JNK phosphorylation was decreased.

\section{Application of the knockdown/knock-in strategy to other selenoproteins}

The strategy of targeting the knockdown of TR1 within its $3^{\prime}$-UTR followed by its replacement worked exceptionally well with this selenoprotein. To determine if this approach can be applied to other selenoproteins, we examined GPx1, which is one of the most widely studied selenoproteins (e.g., see Sunde 2006), and SPS2, which is responsible for generating the active selenium donor, selenophosphate, for Sec synthesis in eukaryotes (see $\mathrm{Xu}$ et al. 2007b, and references therein). The targeting site within the $3^{\prime}$-UTR of GPx1 mRNA that was most effective in its knockdown (see Materials and Methods) was used for further study. NIH3T3 cells were stably transfected with the siGPx1 construct or the pU6-m3 control construct (Fig. 5A,B). siGPx1 stably transfected cells were then transiently transfected with constructs containing either the empty vector (pcDNA), the wild-type GPx1 gene (GPx1 wt), or the GPx1 gene encoding the mutant targeted site (GPxl-in) and the mRNA levels analyzed by Northern blotting (Fig. 5A, lanes 2-4, respectively). GPX1 mRNA was knocked down efficiently as compared to cells encoding the pU6-m3 control construct (Fig. 5A, lanes 1 and 2), and the level of knockdown was the same as that in GPx1 mRNA knockdown observed in cells stably transfected with siGPxl construct alone (data not shown). Cells transiently transfected with the GPx1 wild-type construct showed that the GPx1 mRNA was smeared and reduced compared to cells
A

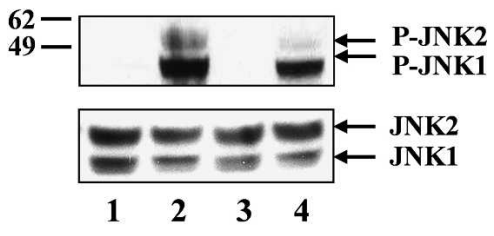

B

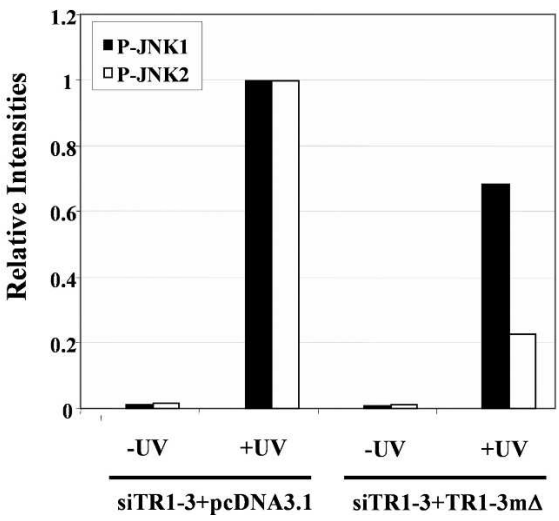

FIGURE 4. UV sensitivity of TR1 replacement cells. (A) Cells stably transfected with siTR1-3 constructs were transiently transfected with the control (pcDNA3.1) or TR1 expression vector (TR1-3m $\Delta$ ), and $24 \mathrm{~h}$ after exposure to UV irradiation, phosphorylation of JNK1 and JNK2 (designated P-JNK1 and P-JNK2) were analyzed by Western blotting (upper panel). (Lanes 1,2) siTR1-3 + pcDNA3.1 untreated and treated with UV irradiation, respectively; (lanes 3,4) siTR1-3 + TR $1-3 \mathrm{~m} \Delta$ untreated and treated with UV irradiation, respectively. Total amounts of JNK are shown as a quantitative control in the lower panel. (B) Intensities of bands relative to P-JNK1 and P-JNK2 in siTR1-3 + pcDNA3.1 UV-treated cells when the latter two bands were given a value of 1.0 and the data plotted as shown. Band intensities were assessed using the ImageQuant program.

transfected with the control construct (Fig. 5A, lanes 1 and 3), while cells transiently transfected with the mutant GPx1 construct manifested a considerable enrichment in GPX1 mRNA (Fig. 5A, lane 4). Labeling each of the cell lines with ${ }^{75} \mathrm{Se}$ and subsequent electrophoresis of protein extracts showed that GPx1 was poorly expressed in GPx1 mRNA knockdown cells (Fig. 5B, cf. lanes 2,3 and lane 1), while GPx1 levels were markedly enriched in the knock-in cells compared to control cells (Fig. 5B, lanes 4 and 1).

The Tet-on system was used for exploring whether SPS2 could be a candidate for the knockdown/knock-in technology. NIH3T3 cells stably transfected with the Tet-siSPS2 construct were grown in the presence or absence of doxycycline. The knockdown vector expressed in the presence of doxycycline targeted the removal of SPS2 mRNA (data not shown). NIH3T3 cells stably transfected with the SPS2 knockdown construct were then transiently transfected with either the wild-type SPS2 construct (SPS2 wt), the knock-in SPS2 construct (SPS2 in), or the control 
A

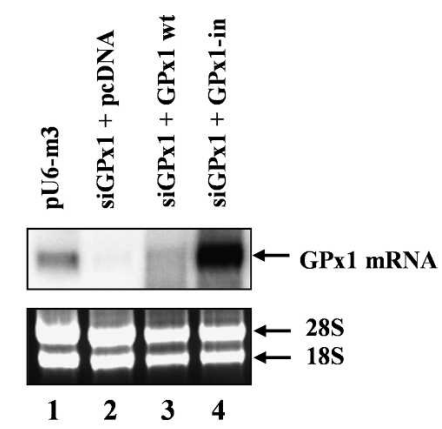

B

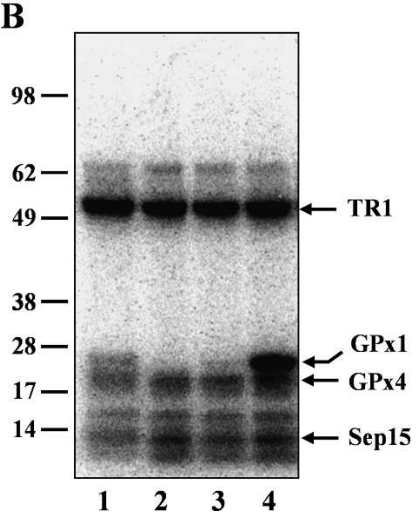

$\mathbf{C}$

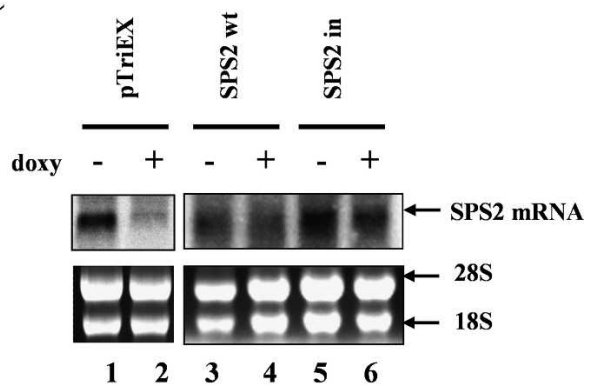

D

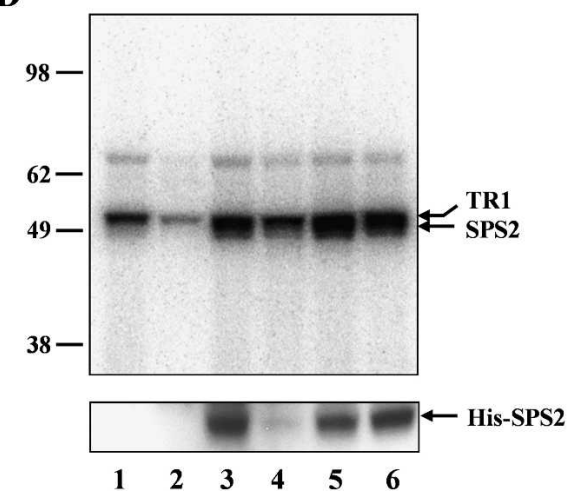

FIGURE 5. Knockdown/knock-in of GPx1 and SPS2. In $A$ and $B$, NIH3T3 cells were stably transfected with the pU6-m3 control construct or the siGPx1 construct and then transiently transfected with the pcDNA3.1 expression vector or the expression vector encoding GPx1 wildtype gene (GPxl wt) or GPxl knock-in gene (GPxl in) and the cells analyzed by $(A)$ Northern blotting of RNA extracts for GPx1 mRNA: (lane 1) pU6-m3 + pcDNA3.1; (lane 2) siGPx1 + pcDNA3.1; (lane 3) siGPx1 + GPx1 wt; and (lane 4) siGPx1 + GPx1-in (upper panel), and (B) labeling of cells with ${ }^{75} \mathrm{Se}$ and subsequent electrophoresis of protein extracts. Lanes $1-4$ are the same as those given in $A$ above. In $C$ and $D$, NIH3T3 cells were stably transfected with the Tet-on U6 control construct or the Tet-siSPS2 construct and grown in the presence or absence of $5 \mu \mathrm{g} / \mathrm{uL}$ doxycycline for $3 \mathrm{~d}$ to induce knockdown SPS2, then transiently transfected with pTriEX expression vector or the expression vector encoding SPS2 wild-type gene (SPS2 wt) or SPS2 knock-in gene (SPS2 in) and the cells analyzed by (C) Northern blot analysis of RNA extracts for SPS2 mRNA: lanes 1 and 2 contain extracts of Tet-siSPS 2 transfected with expression vector in the presence or absence of doxycycline; lanes 3 and 4 contain extracts of Tet-siSPS2 transfected with SPS2 wild-type expressing vector in the presence or absence of doxycycline; lanes 5 and 6 contain extracts of Tet-siSPS2 transfected with the SPS2 wild-type expressing vector in the presence or absence of doxycycline (upper panel) and $(D)$ labeling cells with ${ }^{75} \mathrm{Se}$ and subsequent gel electrophoresis of protein extracts. Lanes 1-6 are the same cell lines as those given in $C$ above. The ${ }^{75}$ Se-labeled panel shows TR1 and the overexpressed SPS2 bands that have similar migrations except SPS2 runs slightly lower. The expression of SPS2 was confirmed by Western blot analysis using antibodies to His-tag of the ectopic SPS2 protein (lower panel). $18 \mathrm{~S}$ and $28 \mathrm{~S}$ ribosomal RNAs are shown as loading controls in the lower panels of $A$ and $C$.

slightly slower on polyacrylamide gels compared to the corresponding, untagged protein. TR1 and SPS2 migrate at approximately the same position on polyacrylamide gels as shown in Figure $5 \mathrm{D}$. As it was difficult to distinguish SPS2 expression independently of TR1 expression in ${ }^{75} \mathrm{Se}$-labeled cells due to their close migration on gels, the expression of SPS2 was further examined by Western blot analysis using antibodies against the His-tag (Fig. 5D, lower panel). SPS2 was clearly expressed in cells carrying the knock-in construct (Fig. 5D, lanes 5 and 6) and in cells carrying the SPS2 wt construct grown in the absence of doxycycline, but poorly expressed in corresponding cells grown in the presence of doxycycline (Fig. 5D, lanes 3 and 4, respectively). The virtual loss of selenoprotein expression due to the knockdown of SPS2, as assessed by ${ }^{75}$ Se labeling (Fig. 5D, lane 2), would be expected, as its removal results in a loss of selenophosphate production, which is essential to selenoprotein synthesis (Xu et al. 2007a). It should be noted that in order to visualize the effect of siSPS2 on SPS2 expression, polyacrylamide gels containing labeled selenoproteins were run much longer to partially separate TR1 and SPS2, which migrate at similar positions. As a consequence, the lower molecular weight selenoproteins ran off the gel (see Fig. 5D). However, the effect of SPS2 knockdown on the entire population of selenoproteins following their labeling with ${ }^{75} \mathrm{Se}$ and separation on polyacrylamide gels can be seen in studies published elsewhere (Xu et al. 2007a). construct (pTriEX), and grown with or without doxycycline. SPS2 mRNA was examined in cell extracts by Northern blotting (Fig. 5C). Clearly, SPS2 was restored in cells carrying the knock-in construct (Fig. 5C, cf. lanes 5,6 and lanes 1,2), but not in cells carrying the SPS2 wt construct grown in the presence of doxycycline (Fig. 5C, lane 4). As expected, cells carrying the SPS2 wt construct grown in the absence of doxycycline had SPS2 mRNA. SPS2 was overexpressed in these cells and therefore was detectable by ${ }^{75} \mathrm{Se}$ labeling. The SPS 2 construct contained a His-tag at its carboxy terminus and therefore it migrated

\section{DISCUSSION}

A major limitation in using RNAi technology to knock down protein expression has been that the resulting phenotype and protein function are assessed solely on the basis of protein loss. This approach does not permit an indepth examination of the characteristics of protein function, for example, by mutational analyses. Assessing protein function by introducing transgenes encoding mutant forms of the protein of interest into a cell also has the limitation of maintaining the host wild-type protein. However, using 
RNAi technology to initially target knockdown of a host protein and then knock-in with a mutant or wild-type protein permits analyses of other aspects of protein function without interference from the host protein as discussed in the Introduction. Selenoproteins would seem particularly amenable to lending themselves to an approach of knockdown/knock-in technology to study their function, as they possess highly developed $3^{\prime}$-UTRs that can be targeted for knockdown of selenoprotein mRNA without affecting the open reading frame of the coding sequence. We have developed such a technology specifically designed for knockdown of selenoproteins by targeting the 3'-UTR. Efficient knockdown of mRNA and protein expression of TR1 were carried out by using two siRNAs that targeted sites downstream of the SECIS element. It is not surprising that TCMK-1 cells stably transfected when these siRNA grew slower than the corresponding control cells carrying wild-type TR1 since TR1 is an essential selenoprotein.

Cells in which the TR1 was stably knocked down were found to be about twice as sensitive to UV irradiation as control cells containing wild-type TR1 activity. In addition, the loss of TR1 expression resulted in cells showing greater signs of UV-mediated apoptosis as evidenced by cleavage of caspase- 3 and an enhanced phosphorylation of JNK1/2. We also previously used RNAi technology to knock down TR1 expression in a mouse lung cancer cell line (LLC1), which reversed several of the malignant phenotypes, including reducing tumor progression and metastasis (Yoo et al. 2006). The alterations in phenotype of TCMK-1 as a result of reduction of TR1 described herein, however, are different from those observed in the LLC1 cell line.

TCMK-1 cells that were stably transfected with the knockdown construct were further transiently transfected with the expression knock-in construct, TR1-3m $\Delta$. Efficient TR1 replacement required, in addition to the mutations at the target site, deletion of the ARE region near the $3^{\prime}$ terminus of the $3^{\prime}$-UTR. The fact that removal of the ARE region is essential for TR1 knock-in was anticipated, since it has been shown that deletion of this region is essential for TR1 overexpression (Gasdaska et al. 1999). Although the level of transient transfection in TR1 replacement cells was $\sim 40 \%$, the amount of TR1 was similar to that observed in control cells as determined by enzymatic activity and Western blotting, but slightly higher as determined by ${ }^{75} \mathrm{Se}$ labeling. The higher TR1 expression levels in cells with only $\sim 40 \%$ transfection levels is most certainly due to the overexpression of TR1, whose gene was cloned into the pcDNA3.1 mammalian expression vector.

UV sensitivity of the TR1 replacement cells showed that cells stably transfected with the knockdown construct and transiently transfected with TR1-3m $\Delta$ were more resistant than their counterpart cells transiently transfected with the pcDNA3.1 vector, suggesting that TR1 replacement restored UV resistance.
The UGA Sec codon in TR1 is the penultimate codeword in the corresponding mRNA. It is possible that the location of the UGA codon in TR1 would make this selenoprotein amenable to knockdown/knock-in technology whereas the UGA Sec codons in most other selenoproteins, which are located elsewhere in sequences, could be less favorable to application of such technology. We therefore examined two other mammalian selenoproteins, GPx1 and SPS2, that contain their Sec residues more proximal to the aminoterminal regions. These two selenoproteins were also selected for study because GPx1 is one of the most widely studied selenoproteins (e.g., see Sunde 2006), and SPS2 plays an essential role in Sec biosynthesis by generating selenophosphate, which is the selenium donor for this pathway (see Xu et al. 2007a,b, and references therein). Targeting sites in the $3^{\prime}$-UTR of both these selenoproteins that effectively knocked down both mRNAs, and the corresponding proteins, were found and further experimentally verified in the knockdown/knock-in procedure.

One of the major criticisms of RNAi technology in assessing the phenotypes associated with knocking down protein expression has been that it may result in off-targeting, wherein another RNA is also affected in addition to the targeted mRNA (Birmingham et al. 2006). Targeting more than a single site, as we have done with the three selenoproteins examined in the present study, substantially decreases the likelihood of off-targeting. The knockdown/knock-in approach, however, eliminates this possibility altogether and should be extremely useful for phenotypic analyses.

Knockdown/knock-in technology provides a new approach to examining many subtleties involving selenoprotein function. For example, a more detailed analyses of Sec UGA codon-nucleotide context (McCaughan et al. 1995), Sec UGA codon-SECIS element association (Korotkov et al. 2002), and a recently identified novel RNA structure present downstream of Sec UGA codons in certain selenoprotein mRNAs (Howard et al. 2005), could be more readily examined by a knockdown/knock-in strategy. Similarly, this approach could presumably be adapted to many other classes of proteins. Clearly, utilizing target sites in the 3'-UTR for developing knockdown/ knock-in strategies has the distinct advantage of avoiding the base changes within the open reading frame that, even though they may not cause an amino acid change, may result in dramatically altering expression of the resulting protein (Kimchi-Sarfaty et al. 2007).

\section{MATERIALS AND METHODS}

\author{
Materials \\ ${ }^{75} \mathrm{Se}$ (specific activity, $1000 \mathrm{Ci} / \mathrm{mmol}$ ) was obtained from the \\ Research Reactor Facility, University of Missouri, Columbia, \\ Missouri, and $\left[\alpha-{ }^{32} \mathrm{P}\right] \mathrm{CTP}$ (specific activity $6000 \mathrm{Ci} / \mathrm{mmol}$ ) from \\ Perkin-Elmer. Primers containing a loop for siRNA hairpin
}


formation, sense, and anti-sense sequences corresponding to the target regions in mouse TR1, GPx1, and SPS2 were prepared by Sigma Genosis, and were designed to have BamHI and HindIII restriction sites at the $5^{\prime}$ and $3^{\prime}$ ends, respectively. pcDNA3.1 mammalian expression vector was purchased from Invitrogen Life Technologies and siRNA vector pSilencer 2.1-U6 Hygro from Ambion, Inc. Antibodies against JNK, phospho-JNK and caspase3 , and HRP-conjugated secondary antibodies were purchased from Cell Signaling Technology and anti-His antibodies from Qiagen. Mouse kidney cells and mouse fibroblast, designated TCMK-1 and NIH3T3, were obtained from the American Type Culture Collection (ATCC). All other reagents were commercial products of the highest grade available.

\section{Knockdown of TR1, GPx1, and SPS2}

pU6-m3 was prepared (Xu et al. 2007a) and used as a cloning vector for siTR1 targeting sequences and to transfect TCMK-1 cells as a control vector. The $3^{\prime}$-UTR of mouse TR1, GPx1, and SPS2 mRNAs (GenBank Accession Numbers NM_015762, NM 008160, and NM 009266, respectively) was surveyed using the diDESIGN program (Dharmacon, Inc.). Four candidate target regions within the $3^{\prime}$-UTR of TR1 were selected that spanned nucleotides 1721-1741, 1760-1780, 1993-2014, and 2480-2500, respectively. Sense-anti-sense oligonucleotides, designed according to the instructions detailed in the cloning manual of the pSilencer 2.1U6 Hygro vector, were annealed and inserted into the BamHI-HindIII cloning sites in pU6-m3. The sequences of the resulting constructs, designated siTR1-1, siTR1-2, siTR1-3, and siTR1-4, were confirmed by sequencing.

For knocking down GPx1, three candidate siRNA targeting sites within the $3^{\prime}$-UTR were selected that spanned nucleotides 702-720, 788-806, and 803-821, and the corresponding knockdown constructs were prepared in the same manner as the TR1 knockdown constructs. Northern blot and ${ }^{75}$ Se-labeling analysis of NIH3T3 cells stably transfected cells with the siRNA constructs demonstrated that the targeting site spanning nucleotides 803-821 was the most effective in knocking down GPx1, and this knockdown construct was used in the present studies as described in the Results.

To knock down SPS2, the doxycyclin-inducible Tet-on U6 construct was prepared using the pU6-m4 Hygro vector (Xu et al. 2007a) as backbone. Two O2-type Tet operators were then generated that flanked the TATA box of the U6 promoter (Lin et al. 2004) using the QuikChange II Site-Directed Mutagenesis Kits, and the resulting vector was designated pU6-TetO4m4. The SPS2 siRNA constructs of pU6-TetO4m4 were prepared as described (Xu et al. 2007a). Of the three separate 19 nucleotide (nt) siRNA target sequences generated within the 3' -UTR of SPS2, Northern blot analysis showed that targeting mRNA spanning1821-1839 was the most effective at knocking down the corresponding mRNA and it was used in subsequent experiments. In SPS2 knockdown and knock-in experiments, cells stably transfected with SPS2 or control siRNA constructs were induced with $5 \mu \mathrm{g} / \mathrm{mL}$ doxycycline for $3 \mathrm{~d}$, then labeled with ${ }^{75} \mathrm{Se}$ or harvested for RNA preparation.

\section{Replacement of TR1, GPx1, and SPS2}

The entire TR1 gene containing both the $5^{\prime}$ - and $3^{\prime}$-UTRs was amplified by RT-PCR of TCMK-1 total RNA and cloned into the pcDNA3.1 mammalian expression vector using the HindIII and XhoI restriction sites to knock in TR1. Mutations in the siRNA target region involving bases 1993-2014 were introduced by PCR using mutant primers, siTR1-3m forward, 5'-gtcttagtctcaaggtacctatgtc taatgtc- $3^{\prime}$, and siTR1-3m reverse, $5^{\prime}$-gacattagacataggtaccttgagactaa gac- $3^{\prime}$, where the bold, lowercase letters indicate mutated regions. Two partial TR1 fragments encompassing bases 15-2014 (from the 5'-UTR to the siRNA target region) and 1993-3310 (from the siRNA target region to the end of the $3^{\prime}$-UTR) were synthesized with mutant primers by PCR, and the full-length TR1 gene was synthesized by annealing the two partial fragments and extending both ends using Pfx DNA polymerase. The product was cloned into pcDNA3.1 (the resulting mutant construct was designated TR1$3 \mathrm{~m}$ ). The AU-rich mRNA instability element (ARE) located in the 3'-UTR of TR1 (bases 2407-3310; Gasdaska et al. 1999) was deleted using BlpI and XhoI restriction sites to enhance the stability of TR1 mRNA, and the resulting mutant construct was designated TR1-3m $\Delta$.

To knock in GPx1 and SPS2, mutations in the target regions in GPx1 (nucleotides 803-821, 5' -gcgagagatgggttcaata-3') and SPS2 (nucleotides 1821-1839, 5' -caagagttgccacaggata- $3^{\prime}$ ) were introduced by PCR where the bold, lowercase letters indicate mutated nucleotides.

\section{Mammalian cell culture, transfection, transient transfection efficiencies, and UV irradiation}

TCMK-1 or NIH3T3 cells were grown in Dulbecco's modified Eagle's medium (DMEM) supplemented with $10 \%$ fetal bovine serum and antibiotic-antimycotic solution at $37^{\circ} \mathrm{C}$ as described in ATCC's product description packet. Cells were maintained in growth phase by splitting every $3 \mathrm{~d}$ using Trypsin-EDTA. Stably transfected siTR1-3 (knockdown) and stably transfected pU6-m3 (control) cells were prepared by transfecting with the corresponding constructs using Lipofectamine 2000 and then selecting cells in the presence of $500 \mu \mathrm{g} / \mathrm{mL}$ of hygromycin B. In addition, both these stably transfected cell lines were transiently transfected with the control (pcDNA3.1), wild-type (TR1wt), and mutant (siTR1$3 \mathrm{~m}$ and siTR1-3ms) constructs. Efficiency of transient transfection was measured by cotransfecting GFP expression construct with the pcDNA3.1 or TR1-m3s constructs, counting GFP expressing cells under a fluorescent microscope, and was found to be $\sim 40 \%$ in each case (data not shown).

To assess the UV sensitivity of each cell line, $1.0 \times 10^{6}$ cells were seeded onto a $60 \mathrm{~mm}$ culture dish, and, in the case of transiently transfected cells, the cells were seeded $24 \mathrm{~h}$ after transfection, and each cell line incubated $24 \mathrm{~h}$ before irradiating with $\mathrm{UV}\left(30 \mathrm{~J} / \mathrm{m}^{2}\right)$; UV-irradiated cells then harvested at preselected times (see legends to figures) for assessing JNK phosphorylation and caspase-3 cleavage. Cells were harvested and counted using the Trypan-blue exclusion method (Freshney 1987) or assessed for JNK phosphorylation and caspase- 3 cleavage by Western blotting $24 \mathrm{~h}$ after irradiation. The relative amounts of JNK phosphorylation following Western blot analysis were quantitated using the ImageQuant program.

\section{Northern and Western blot analyses, ${ }^{75}$ Se labeling of cells, and assay for thioredoxin reductase activity}

Total RNA was prepared from cultured cells, $12 \mu \mathrm{g}$ of total RNA electrophoresed and transferred, membranes hybridized with probes for TR1, GPx1, or SPS2 (prepared by random labeling with $\left[\alpha-{ }^{32} \mathrm{P}\right] \mathrm{CTP}$ ), 
washed, and exposed to a PhosphorImager (Molecular Dynamics) as described (Moustafa et al. 1998; Carlson et al. 2005).

Cells were washed in cold PBS, lysates prepared, $30 \mu \mathrm{g}$ of each protein sample electrophoresed, the separated proteins transferred, and the membrane incubated initially with primary antibody (antiphospho-JNK, anti-JNK, anti-caspase-3, or anti-His) and then with HRP-conjugated secondary antibody and exposed to X-ray film as described (Moustafa et al. 1998; Carlson et al. 2005).

Cells were seeded onto a six-well plate $\left(3 \times 10^{5}\right.$ cells/well $)$, incubated $18 \mathrm{~h}$, then labeled with $40 \mu \mathrm{Ci}$ of ${ }^{75} \mathrm{Se}$ for $24 \mathrm{~h}$, and harvested and lysed as described (Moustafa et al. 1998; Carlson et al. 2005). Forty micrograms of each sample were electrophoresed, proteins stained with Coomassie Blue, and the gel dried and exposed to a PhosphorImager (Molecular Dynamics) as described (Moustafa et al. 1998; Carlson et al. 2005).

The specific enzymatic activity of TR in cell extracts was determined using the insulin assay as described (Arner et al. 1999).

\section{ACKNOWLEDGMENTS}

This research was supported by the Intramural Research Program of the National Institutes of Health, National Cancer Institute, Center for Cancer Research, and by NIH GM065204 and GM061603 grants (V.N.G.).

Received March 2, 2007; accepted March 26, 2007.

\section{REFERENCES}

Arner, E.S., Zhong, L., and Holmgren, A. 1999. Preparation and assay of mammalian thioredoxin and thioredoxin reductase. Methods Enzymol. 300: 226-239.

Birmingham, A., Anderson, E.M., Reynolds, A., Ilsley-Tyree, D., Leake, D., Fedorov, Y., Baskerville, S., Maksimova, E., Robinson, K., Karpilow, J., et al. 2006. 3'-UTR seed matches, but not overall identity, are associated with RNAi off-targets. Nat. Methods 3: 199-204.

Carlson, B.A., Xu, X.M., Gladyshev, V.N., and Hatfield, D.L. 2005. Selective rescue of selenoprotein expression in mice lacking a highly specialized methyl group in selenocysteine tRNA. J. Biol. Chem. 280: 5542-5548.

Freshney, R. 1987. Culture of animal cells: A manual of basic techniques. Alan R. Liss, Inc., New York.

Gasdaska, J.R., Harney, J.W., Gasdaska, P.Y., Powis, G., and Berry, M.J. 1999. Regulation of human thioredoxin reductase expression and activity by $3^{\prime}$-untranslated region selenocysteine insertion sequence and mRNA instability elements. J. Biol. Chem. 274: 25379-25385.

Hannon, G.J. 2002. RNA interference. Nature 418: 244-251.

Hatfield, D.L., Berry, M.J., and Gladyshev, V.N., eds. 2006. Selenium: Its molecular biology and role in human health, 2nd ed. Springer Science+Business Media, New York.

Howard, M.T., Aggarwal, G., Anderson, C.B., Khatri, S., Flanigan, K.M., and Atkins, J.F. 2005. Recoding elements located adjacent to a subset of eukaryal selenocysteine-specifying UGA codons. EMBO J. 24: 1596-1607.

Jallow, Z., Jacobi, U.G., Weeks, D.L., Dawid, I.B., and Veenstra, G.J. 2004. Specialized and redundant roles of TBP and a vertebratespecific TBP paralog in embryonic gene regulation in Xenopus. Proc. Natl. Acad. Sci. 101: 13525-13530.

Jiang, Y. and Price, D.H. 2004. Rescue of the TTF2 knockdown phenotype with an siRNA-resistant replacement vector. Cell Cycle 3: $1151-1153$.

Kiang, A.S., Palfi, A., Ader, M., Kenna, P.F., Millington-Ward, S., Clark, G., Kennan, A., O’Reilly, M., Tam, L.C., Aherne, A., et al.
2005. Toward a gene therapy for dominant disease: Validation of an RNA interference-based mutation-independent approach. Mol. Ther. 12: 555-561.

Kim, D.H. and Rossi, J.J. 2003. Coupling of RNAi-mediated target down-regulation with gene replacement. Antisense Nucleic Acid Drug Dev. 13: 151-155.

Kimchi-Sarfaty, C., Oh, J.M., Kim, I.M., Sauna, Z.E., Calcagno, A.M., Ambudkar, S.V., and Gottesman, M.M. 2007. A "silent" polymorphism in the MDR1 gene changes substrate specificity. Science 315: 525-528.

Korotkov, K.V., Novoselov, S.V., Hatfield, D.L., and Gladyshev, V.N. 2002. Mammalian selenoprotein in which selenocysteine $(\mathrm{Sec})$ incorporation is supported by a new form of Sec insertion sequence element. Mol. Cell. Biol. 22: 1402-1411.

Kryukov, G.V., Castellano, S., Novoselov, S.V., Lobanov, A.V., Zehtab, O., Guigo, R., and Gladyshev, V.N. 2003. Characterization of mammalian selenoproteomes. Science 300: 1439-1443.

Laatsch, A., Ragozin, S., Grewal, T., Beisiegel, U., and Joerg, H. 2004. Differential RNA interference: Replacement of endogenous with recombinant low density lipoprotein receptor-related protein (LRP). Eur. J. Cell Biol. 83: 113-120.

Lin, X., Yang, J., Chen, J., Gunasekera, A., Fesik, S.M., and Shen, Y. 2004. Development of a tightly regulated U6 promoter for shRNA expression. FEBS Lett. 577: 376-380.

Low, S.C. and Berry, M.J. 1996. Knowing when not to stop: Selenocysteine incorporation in eukaryotes. Trends Biochem. Sci. 21: 203-208.

McCaughan, K.K., Brown, C.M., Dalphin, M.E., Berry, M.J., and Tate, W.P. 1995. Translational termination efficiency in mammals is influenced by the base following the stop codon. Proc. Natl. Acad. Sci. 92: 5431-5435.

Moustafa, M.E., El-Saadani, M.A., Kandeel, K.M., Mansur, D.B., Lee, B.J., Hatfield, D.L., and Diamond, A.M. 1998. Overproduction of selenocysteine tRNA in Chinese hamster ovary cells following transfection of the mouse tRNA ${ }^{[\mathrm{Ser}] \mathrm{Sec}}$ gene. RNA 4: 1436-1443.

Mustacich, D. and Powis, G. 2000. Thioredoxin reductase. Biochem. J. 346: $1-8$.

Novina, C.D. and Sharp, P.A. 2004. The RNAi revolution. Nature 430: $161-164$.

Puthenveedu, M.A. and Linstedt, A.D. 2004. Gene replacement reveals that p115/SNARE interactions are essential for Golgi biogenesis. Proc. Natl. Acad. Sci. 101: 1253-1256.

Rundlof, A.K. and Arner, E.S. 2004. Regulation of the mammalian selenoprotein thioredoxin reductase 1 in relation to cellular phenotype, growth, and signaling events. Antioxid. Redox Signal. 6: 41-52.

Sunde, R.A. 2006. Regulation of glutathione peroxidase-1 expression. In Selenium: Its molecular biology and role in human health, 2nd ed. (eds. D.L. Hatfield et al.), pp. 149-160. Springer Science+Business Media, New York.

Unwalla, H.J., Li, H.T., Bahner, I., Li, M.J., Kohn, D., and Rossi, J.J. 2006. Novel Pol II fusion promoter directs human immunodeficiency virus type 1-inducible coexpression of a short hairpin RNA and protein. J. Virol. 80: 1863-1873.

Xu, X.M., Carlson, B.A., Irons, R., Mix, H., Zhong, N., Gladyshev, V.N., and Hatfield, D.L. 2007a. Selenophosphate synthetase 2 is essential for selenoprotein biosynthesis. Biochem. J. 404: $115-120$.

Xu, X.M., Carlson, B.A., Mix, H., Zhang, Y., Saira, K., Glass, R.S., Berry, M.J., Gladyshev, V.N., and Hatfield, D.L. 2007b. Biosynthesis of selenocysteine on its tRNA in Eukaryotes. PLoS Biol. 26: 96-105.

Yoo, M.H., Xu, X.M., Carlson, B.A., Gladyshev, V.N., and Hatfield, D.L. 2006. Thioredoxin reductase 1 deficiency reverses tumor phenotype and tumorigenicity of lung carcinoma cells. J. Biol. Chem. 281: 13005-13008.

Yu, Q., Sicinska, E., Geng, Y., Ahnstrom, M., Zagozdzon, A., Kong, Y., Gardner, H., Kiyokawa, H., Harris, L.N., Stal, O., et al. 2006. Requirement for CDK4 kinase function in breast cancer. Cancer Cell 9: 23-32. 

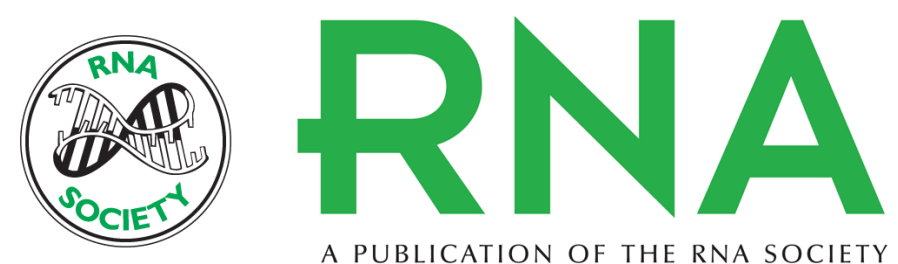

A PUBLICATION OF THE RNA SOCIETY

\section{A new strategy for assessing selenoprotein function: siRNA knockdown/knock-in targeting the 3 '-UTR}

Min-Hyuk Yoo, Xue-Ming Xu, Anton A. Turanov, et al.

RNA 2007 13: 921-929 originally published online April 27, 2007

Access the most recent version at doi:10.1261/rna.533007

$\begin{array}{ll}\text { References } & \begin{array}{l}\text { This article cites } 27 \text { articles, } 14 \text { of which can be accessed free at: } \\ \text { http://rnajournal.cshlp.org/content/13/6/921.full.html\#ref-list-1 }\end{array}\end{array}$

License

Email Alerting Receive free email alerts when new articles cite this article - sign up in the box at the top Service right corner of the article or click here.

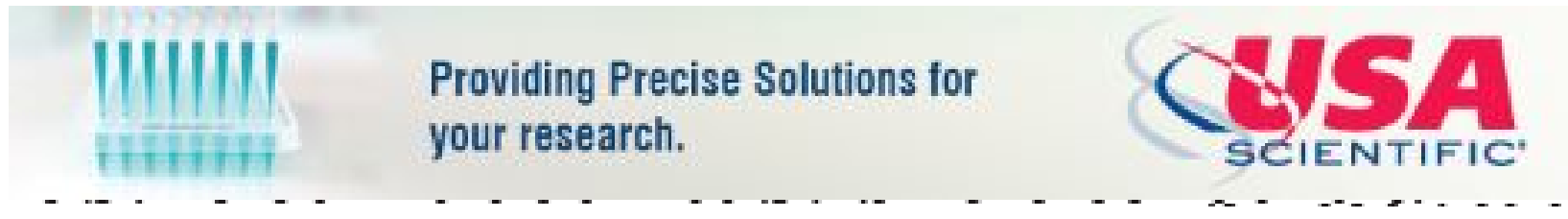

To subscribe to $R N A$ go to:

http://rnajournal.cshlp.org/subscriptions 